\title{
Antibiotic use associated to decreased recognition of facial emotion expressions and increased aggressive cognitive reactivity: a preliminary study
}

\author{
Anne C. D. de $\operatorname{Groot}^{1} \&$ Laura Steenbergen ${ }^{1}$ \\ ${ }^{1}$ Leiden University, Cognitive Psychology Unit \& Leiden Institute for Brain and Cognition, Leiden, the \\ Netherlands
}

Correspondence concerning this article should be addressed to Dr. Laura Steenbergen, Cognitive Psychology Unit, Leiden University, Wassenaarseweg 52, 2333AK Leiden, the Netherlands. Contact: L.Steenbergen@fsw.leidenuniv.nl

\begin{abstract}
The microbiota-gut-brain axis has been proposed a key regulator of the stress response, providing possibilities for the prevention and treatment of stress-related disorders. Manipulations of the gut microbiome seem to exert psychological effects affecting cognitive- and emotional reactivity. We hypothesized antibiotics to increase cognitive reactivity to sad mood and impair the recognition of emotions. Thirty-four Dutch participants participated in this study, of which 16 finished antibiotic treatment in the month prior to testing (i.e. the antibiotic group); the remaining 18 reported no antibiotic use in the past year (i.e. the control group). We assessed self-reported mood, cognitive reactivity to sad mood, and depression, as well as accuracy in recognizing facial emotional expressions. The antibiotic group demonstrated increased cognitive reactivity to sad mood, specifically aggressive thoughts, but did not differ from controls on measures of mood or depressive symptoms. In addition, the antibiotic group demonstrated lower accuracy in recognizing moderate intensity emotions and fear in general. This study provides preliminary evidence associating antibiotic use and cognitive reactivity to sad mood, specifically aggression, as well as recognizing emotions, supporting the idea that the microbiota-gutbrain axis modulates cognitive- and emotional reactivity. Further research is needed to confirm, and gain understanding of, these effects.
\end{abstract}




\section{Introduction}

A growing line of research indicates brain functioning and behavior might be modulated by the bidirectional communication between gut microbiota, referring to all microorganisms colonizing the intestines, and the brain (i.e. the microbiota-gut-brain axis; Bercik and Collins, 2014; Cryan and Dinan, 2012; Cryan and O'Mahony, 2011; Forsythe et al., 2016; Foster and McVey-Neufeld, 2013; Luna and Foster, 2015; Wallace and Milev, 2017). Gut microbiota may be viewed as an organ; exerting metabolic functions as well as regulating inflammatory responses by immune activation. Disturbances in the homeostasis of the gut microbiota, for example as a consequence of antibiotics, result in alterations at neural, hormonal, and immunological levels (Cryan and O'Mahony, 2011; Cryan and Dinan, 2012; Delungahawatta et al., 2017; Dudek-Wicher et al., 2018; Foster and McVey-Neufeld, 2013; Lurie et al., 2015; Sherwin et al., 2018). Interestingly, such alterations have been found to associate to changes in the physiological stress-response (e.g. activation of the stress axis) and associated behavior (e.g. sociability and avoidance; Sherwin et al., 2018) in both animals (Foster and McVey-Neufeld, 2013; Leclercq et al., 2017; McVey-Neufeld et al., 2011; Rhee et al., 2009) and humans (Messsaoudi et al., 2011; Steenbergen et al., 2015). However, the cognitive- and affective consequences of microbiota manipulations remain under investigated; "psychologists have yet to join this journey" (Sarkar et al., 2018 p. 1).

Not unsurprisingly in light of the preceding, a body of research demonstrated associations between the disturbed or dysbiosis in the gut microbiota and psychiatric stress- and emotion-related disorders, such as depression (e.g., Sternbach and State, 1997), anxiety (e.g., Jiang et al., 2018), and autism (e.g., Iovene et al., 2017), but also schizophrenia (Castro-Nallar et al., 2015) and Parkinson's disease (Lin et al., 2018), for a review see Rieder et al. (2017) and Sarkar et al (2018). Subsequently, interventions targeted at manipulating the gut microbiota, for example by means of probiotics, demonstrated a possible causal role of the gut microbiome in stress- and emotion regulation (Messaoudi et al., 2011; Papalini et al., 2019; Steenbergen et al., 2015). Such studies are typically targeted at reducing symptom severity and enhancing subjective wellbeing. However, the aforementioned disorders all have in common that they are associated to dysfunctions in cognitive- and affective processes, most 
importantly emotional processing; functions which are found predictive of such disorders (e.g. Turetsky et al., 2007; Harmer, 2008). This illustrates the need for investigating the effects of microbiota-gut-brain manipulations regarding emotional processing, which is further supported by the suggestion that antidepressants may not directly modulate mood and anxiety, but instead induce changes in emotional processing that precede and allow, but not necessarily evoke, changes in subjective outcomes (Harmer, 2008).

In addition, measurements of emotional processes (e.g. recognition of facial emotional expressions) are more sensitive than self-reported mood measurements (Harmer, 2008), and provide a gateway to study the cognitive effects of psychobiotics in otherwise healthy individuals. Regarding cognitive- and emotional processing in relation to manipulations of the gut microbiota, Steenbergen and collegues (2015) reported that a 4-week probiotic intervention resulted in reductions in aggressive and ruminative thought patterns in response to a sad mood (i.e. cognitive reactivity). Additionally, as a possible explanation of such effects, Sarkar et al. (2018) suggest psychobiotics may reduce emotional reactivity. As rightfully noted by the authors, this would also result in decreased reactivity to positive stimuli. Indeed, in a functional magnetic resonance imaging study by Tillisch and colleagues (2013), activity of a brain network central to processing emotions during an emotion recognition task was reduced after a 4-week probiotic intervention in healthy women. In contrast, however, Kelly et al. (2017) reported no modifying effects of an 8-week probiotic intervention in healthy males on stressrelated measures (i.e. measures of mood, anxiety, and stress) and cognitive performance (i.e. emotion recognition). Mood and cognitive effects are thus not always observed in probiotic interventions, calling for further investigation of the microbiota-gut-brain axis and cognitive-and affective consequences of its manipulations.

In contrast to probiotics, antibiotics contribute to dysbiosis (i.e. microbial imbalance of intestinal bacteria of the gut microbiome; Nicholson et al., 2012; Preidis and Versalovic, 2009; Zarrinpar et al., 2018). Antibiotics are commonly prescribed for bacterial infections (Foster and McVey-Neufeld, 2013; Preidis and Versalovic, 2009), which shows a steep increase in the past decade (Haeseker et al., 2012). However, antibiotic use has been associated with an increased risk for depression and anxiety (Lurie et al., 2015; Macedo et al., 2017), possibly as a consequence of microbial 
dysbiosis. Indeed, patients suffering depression or anxiety show a reduction in species richness and microbial diversity, indicative of dysregulated microbiota (Sherwin et al., 2018). Although correlational, findings in animal studies support the assumption that dysregulation of the gut microbiota facilitates behavioral (e.g. reduced exploratory behavior and grooming, increased risk assessment) and psychological (e.g. poorer spatial memory, impaired recognition memory) symptoms of depression and anxiety (Sherwin et al., 2018; Sarkar et al., 2018; Fiore et al., 1996; Lyte et al., 2006; Slykerman et al., 2017; Wang et al., 2015). However, a study by Bercik and colleagues (2011) regarding preliminary data of $\mathrm{BALB} / \mathrm{c}$ mice that underwent a seven day antibiotic intervention reported antibiotic treated mice showed a reduction in anxiety through an increase in exploratory (i.e. locomotor) behavior compared to the control group, illustrating the opposite effect. In humans, such evidence is scarce but a recent study suggested that antibiotic treatment (i.e. minocycline) may have antidepressive effects, in this case improvement in global experience, functioning and quality of life in people with major depressive disorder (Dean et al., 2017). However, the consequences of antibiotic use in respect to mental functions and, ultimately, well-being of otherwise healthy individuals remains unknown.

Within the available rodent studies, the cognitive consequences of antibiotic administration remain inconclusive (for a review see Sarkar et al., 2018). Regarding humans, a recent pilot study by Wang et al. ${ }^{40}$ concluded a low dose antibiotic to exhibit stress-reducing effects similar to reported effects of probiotics. More specifically, a significant increase of "emotional well-being", measured using the SF-36, was found in the antibiotic group compared to the placebo group. Taking a closer look at these findings, the antibiotic group improved regarding negative items while remaining the same on the positive items. That is, in line with the suggestion by Sarkar et al. (2018), the authors seem to have observed a reduction in emotional reactivity with no gain regarding positive affect.

The above allows topredict that the microbiota-gut-brain axis may affect cognitive- and affective reactivity, which may precede and allow, but not necessarily evoke, mood effects. The current pilot study therefore aims to further explore such a possibility by investigating the effects of antibiotic usage on cognitive reactivity to sad mood and facial emotion expression recognition ${ }^{24}$, mood, and depression symptoms. However, in addition to the under investigated role of the microbiota-gut-brain axis in cognitive- and affective processing in healthy humans, given the lack of conclusive results 
regarding antibiotic effects on cognitive- and emotional reactivity to stress and subsequent symptoms of depression and anxiety, and the current study being the first to examine the effects of antibiotic use on the ability to recognize facial expressions of emotions, the nature of the current study is rather exploratory. That is, given the findings regarding probiotics and cognitive reactivity to sad mood by Steenbergen et al. (2015) (but see Papalini et al., 2019), we predict opposite effects, i.e. antibiotics to exert a negative effect on cognitive reactivity to sad mood, possibly specifically rumination and aggression.

Regarding the recognition of facial emotion expressions, if antibiotics, in contrast to probiotics (Sarkar et al., 2018), increase emotional reactivity, one might expect increased accuracy in recognizing emotional expressions. However, expecting impairments in recognizing emotional expressions may be more plausible; first, along the lines of the findings by Harmer and colleagues (2001; Harmer, 2008), antibiotics may exert antidepressive effects through impairing one's ability to recognize negative emotional expressions. Seemingly paradoxal, this may simultaneously explain why antibiotics may increase the risk for depression; whereas patients suffering from depression may benefit from a reduction in the ability to recognize facial emotion expressions, especially of negative emotions, the inability to adequately recognize emotions in healthy individuals may contribute to vulnerability to depression. In order to test whether antibiotics reduce one's ability to accurately recognize facial expressions of emotions, we used a Facial Emotion Expression Recognition task (adapted from Harmer et al., 2001). Importantly, responses to facial expressions of anger, fear, sadness, surprise, happiness, and disgust are able to be studied as a function of intensity (i.e. low, moderate or high) of the expressed emotion. Antibiotic use may affect the recognition of moderate expression as they are more sensitive to subtle manipulations (Harmet et al., 2001; Harmer, 2008).

\section{Method}

\section{Participants and Design}

Thirty-four Dutch participants aged 18-55 years (12 males) participated in this study. Participants were recruited through advertisements, word-to-mouth, social media, and an online 
research participation platform; the study was advertised as a one-session study on the relation between antibiotic use and well-being. Specifically, we advertised that we were looking for participants currently taking, or just having finished taking, antibiotic treatment. In keeping with Steenbergen et al. (2015), participants were screened using the Mini International Neuropsychiatric Interview (MINI; Sheehan et al., 1998) and considered eligible for participation if they reported no psychiatric or neurological disorders, no personal or family history of depression or migraine, no cardiac, renal, or hepatic conditions, no allergies or intolerance to lactose or gluten, and no prescribed medication or drug use (with the exception of antibiotics). Participants were tested within 1 day to 2 weeks after finishing prescribed antibiotics, and only planned for testing when they reported no longer experiencing complaints related to the condition they were prescribed antibiotics for. These participants were assigned to the antibiotics group. For each participant included to this group, we recruited one participant for the control group; participants meeting the same criteria but not having taken any antibiotics in the past 6 months. Participants were offered 15 euros or participation credits in return for participation. Written informed consent was obtained from all participants before inclusion, the protocol and procedure conformed to the ethical standards of the 1975 Declaration of Helsinki, as revised in 1983, and were approved by the local ethics committee (Commissie Ethiek Psychologie, University of Amsterdam, \#2017-CEP-8359).

\section{Procedure}

The procedure relevant for the current study was part of a larger study on antibiotic use and well-being, results (i.e. regarding physical consequences of antibiotic use) of which will be reported elsewhere. Hence, only procedures relevant for our hypotheses will be described in detail. All participants were tested individually. Upon arrival, participants read and signed the informed consent, after which they filled out the Positive and Negative Affect Scale (PANAS; Watson et al., 1988), performed the Facial Expression Recognition task (FERT; Harmer et al., 2001), answered the Center for Epidemiology Depression scale (CESD; Radloff, 1977; Bouma et al., 1995), Rumination Response Scale (RRS; Nolen-Hoeksema and Morrow, 1991; Raes et al., 2009a), and the Leiden Index for Depression 
Sensitivity - revised (LEIDS-r; van der Does and Williams, 2003; Solis et al., 2017). Subsequently, participants were debriefed and compensated for participation.

\section{Positive and Negative Affect Scale (PANAS)}

The positive and negative affect scale (PANAS; $\alpha=.90$; Watson et al., 1988) was used to infer current mood state. The PANAS is a validated questionnaire comprising a 10 -item positive mood scale and a 10-item negative mood scale (i.e. 20 in total), developed for the brief measurement of positive and negative affect. Participants indicate how much they experience 10 positive and 10 negative emotions at this moment, on a 5-point likert-type scale ranging from 1 'very slightly or not at all' to 5 'very much'. Total scores on both mood scales range from 10 to 50 and are calculated by summing the respective items.

Leiden Index of Depression Sensitivity - revised (LEIDS-r)

To assess cognitive reactivity to sad mood, we used the revised version of the Leiden Index of Depression Sensitivity (LEIDS-r; van der Does and Williams, 2003) which consists of 34 statements rated on a 5-point likert scale ( $\alpha=.92$, Solis et al., 2017) ranging from 0 'not at all' to 4 'completely applies to me'. This scale assesses the extent to which dysfunctional thought patterns are activated when an individual experiences mild dysphoria (i.e. cognitive reactivity). The LEIDS-r scores are based on a total score ranging from 0 to 136 , with higher scores indicating more cognitive reactivity. Cognitive reactivity is assessed using six subscales: aggression $(\alpha=.80)$, hopelessness/suicidality $(\alpha=.83)$, acceptance/coping $(\alpha=.64)$, control/perfectionism $(\alpha=.66)$, risk aversion $(\alpha=.81)$, and rumination ( $\alpha$ $=.82$; Solis et al., 2017). Before participants answer the statements they are instructed to take a moment to imagine a day in which they felt a bit down, but not depressed. After which participants are asked to indicate to what extend ('good', 'a little' or 'not at all') they are able to imagine such a day.

Rumination Response Scale (RRS)

The Dutch version of the rumination response scale (RRS; Nolen-Hoeksema and Morrow, 1991) comprised of 22 items ( $\alpha=.92$, Raes et al., 2009a) was used to measure rumination. Participants are asked to rate how often they think or do something when they are sad or down on a scale from 1 'never' to 5 'almost always'. In addition to a total score, the RRS also provides subcores for brooding 
(maladaptive rumination, related to depression) and reflection (adaptive rumination, effective problem solving; Raes et al., 2009b).

Center for Epidemiology - Depression scale (CESD)

The center for epidemiology depression scale (CESD; Radloff, 1977) includes 20 statements ( $\alpha$ $=.79-\alpha=.92$, Bouma et al., 1995) that are rated by participants from 0 'never' to 3 'always' and measures depression symptoms of four different categories: somatic-retarded activity, depressed affect, and interpersonal affect. The CESD is only used for research purposes and not in a clinical setting.

\section{Facial Emotion Expression Recognition task}

The computerized 'Facial Emotion Expressions Recognition task' (FERT) was adapted from Harmer and colleagues (2001), and presented male and female morphed facial expressions from the Ekman and Friesen (1976) database using E-Prime 2.0 software (Psychology Software Tools, Inc., Pittsburgh, PA). Five basic facial expressions, i.e. happiness, anger, fear, sadness, and disgust were shown with an emotion intensity ranging from 10 to $100 \%$ in steps of 10 . Participants were first shown 12 'practice' trials (i.e. one of the 5 emotions displayed as a word, or the word 'neutraal'; Dutch for 'Neutral') to familiarize with the response keys. In total, 102 pictures were presented twice in random order (i.e. a total of 204 stimuli); representing the five emotion categories in 10 different intensities expressed by 2 actors (one male, one female), and a neutral expression by the same two actors. Participants had to respond using labeled keys to indicate which of the five basic emotions was presented. Between trials, a central fixation cross was presented for 1 second. The ten expressions of emotion intensities were reduced to three categories, i.e. Low intensity of expression $(10 \%, 20 \%$, and $30 \%)$, moderate intensity $(40 \%, 50 \%, 60 \%$, and $70 \%)$ and high intensity of expression $(80,90 \%$, and $100 \%)$. Previously it was shown that stimuli with a (moderate) intensity $(40-70 \%)$ allow the detection of more subtle impairments and are sensitive to pharmacological manipulations (Labuschagne et al., 2010). In Kemmis et al. (2007) 'Fear' was in most cases confused with 'Surprise'. In the present study, 'Surprise' was therefore excluded to prevent confusion. Dependent variables were percentage accurate responses and corresponding mean latencies for the five basic facial expressions in 3 intensity categories (Kemmis et al., 2007). 


\section{Statistical Analyses}

All analyses were done using IBM SPSS Statistics (version 25.0). For each questionnaire mean group scores (total and/or partial) were calculated and submitted to an independent samples t-test with group (antibiotic vs. controls) as a between-subjects factor. For the hypotheses to be accepted alpha was set to $p=.05$.

Repeated measures analysis of variance (RM ANOVA) with intensity (low vs. medium vs. high) and emotion (anger, sadness, happiness, disgust, and fear) as within-subjects factor and group (antibiotic vs. control) as between-subjects factor was used for the analysis of the facial emotion expression recognition task (accuracy scores and reaction times). Again all alpha levels were set to $p=$ .05. Where applicable, when the sphericity assumption was violated, Greenhouse-Geisser -corrected values and degrees of freedom are reported. Pairwise comparisons (Bonferroni-adjusted post-hoc tests) were performed to clarify mean differences in case of significant interactions.

\section{Results}

Thirty-four participants completed participation in this study, of which 16 (5 males, $M_{\text {age }}=$ 24.7 \pm 2.1 years; $M_{\mathrm{bmi}}=21.5 \pm 0.6$ ) reported to have used antibiotics in the past month, and 18 ( 7 males, $M_{\text {age }}=25.3 \pm 2.4$ years; $M_{\mathrm{bmi}}=21.3 \pm 0.5$ ) reported no antibiotic use in the past 6 months. For the individuals in the antibiotic group, not everyone was able to recall which antibiotics s/he used, but amongst the use that was reported were Isotretion, Sofradex, Azitromycine, and Nitrofurantoine.

\section{Questionnaires}

Despite screening, two participants in the antibiotics group reported to use citalopram (i.e. a commonly prescribed antidepressant), and were hence excluded from further analyses. One participant from the control group did not fill out the LEIDS-r and responses to that questionnaire for this individual were considered missing. Independent samples t-tests revealed a significant difference for the LEIDS-r subscale aggression, $t(29)=-3.622, p=.001$, indicating participants having received antibiotics 
in the past month show more aggressive thought patterns in response to a sad $\operatorname{mood}\left(M_{\text {aggression }}=8.0, S E\right.$ $=0.9)$ compared to individuals that reported no intake of antibiotics $\left(M_{\text {aggression }}=4.1, S E=0.6\right)$. Although participants in the antibiotics group show a trend towards higher scores on all questionnaires, no other between-group differences were found, see Table 1 .

\section{Facial Emotion Expression Recognition}

\section{Accuracy}

Due to technical error, responses of two participants (one in each group) evaluating the facial expressions were not saved, hence considered missing. RM ANOVA performed on the accuracy data of the remaining 30 participants demonstrated four significant sources of variance. First, a main effect of emotion, $F(4,112)=16.639, p<.001, \eta_{\mathrm{p}}{ }^{2}=.373$, and a main effect intensity, $F(1.576,44.121)=25.614, p$ $<.001, \eta_{\mathrm{p}}{ }^{2}=.967$. Furthermore, it yielded a significant interaction between emotion and intensity, $F(5.061,141.696)=14.440, p<.001, \eta_{\mathrm{p}}{ }^{2}=.340$. Bonferroni-adjusted post-hoc tests indicated participants performed worse on accurately recognizing low-intensity compared to moderate- and high-intensity emotions, and better on recognizing high than moderate-intensity emotions for all emotions except disgust; for which no significant difference between accuracy in recognizing moderate and high intensity emotions was observed ( $p=.073$ ), see Table 2 . Last, we observed an interaction between intensity and group, $F(1.576,44.121)=4.584, p=.022, \eta_{\mathrm{p}}{ }^{2}=.141$. Bonferroni-adjusted post-hoc tests indicated recognition accuracy was significantly lower in the antibiotics compared to the control group for moderate intensity emotions $(p=.024)$ but not low $(p=.382)$ or high intensity emotions $(p=.812)$, see Table 2. Although no further effects were observed, pairwise comparisons (Bonferroni adjusted) did demonstrate a significant between-group difference for the accurate recognition of fear $(p=.011$, all other $p_{\mathrm{s}} \geq .250$ ), indicating participants in the antibiotics group were less accurate in recognizing expressions of fear, see Table 2 . 
Table 1. Mean questionnaire scores and standard errors as a function of group (control vs. antibiotics).

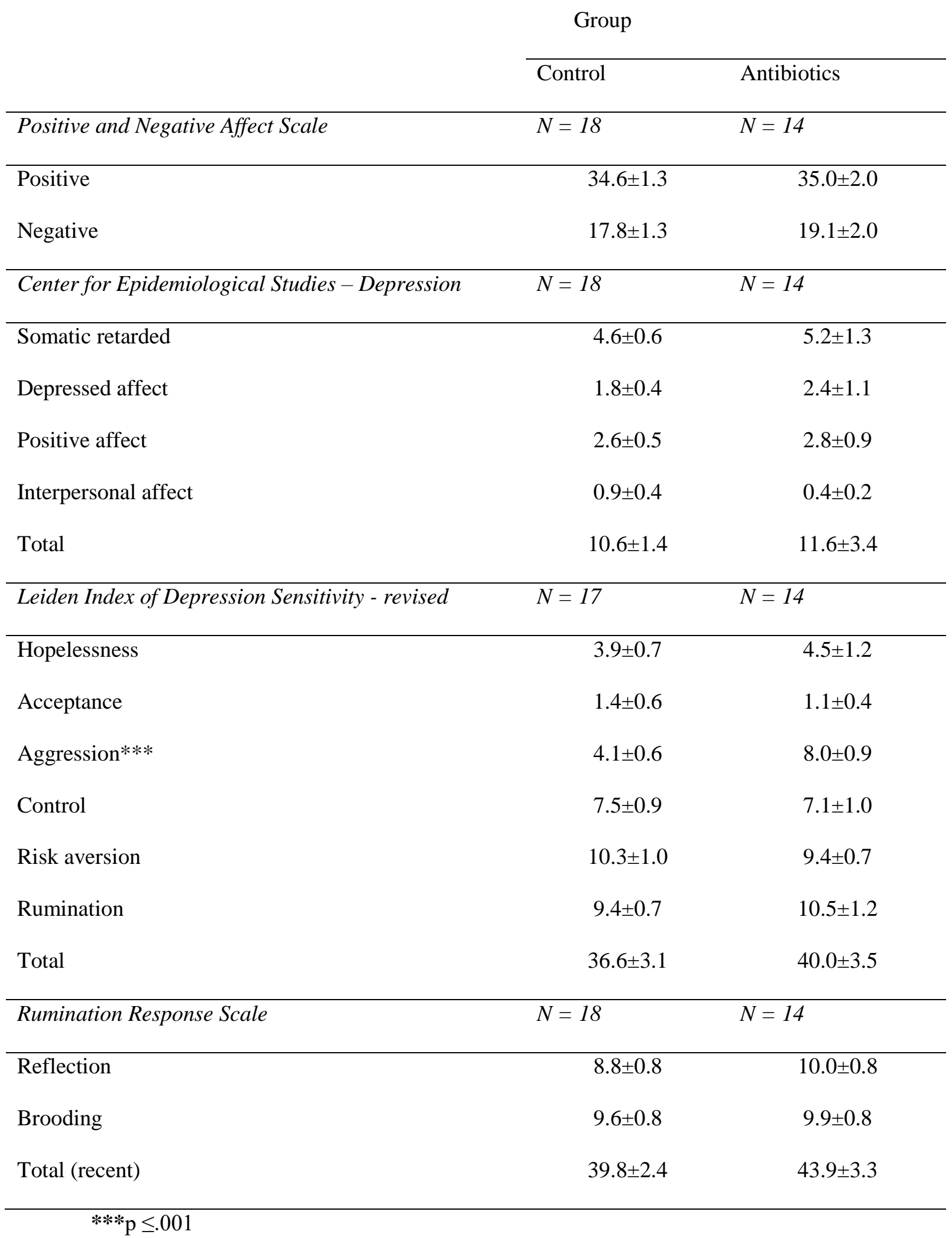


Table 2. Percentage accurate responses to recognizing facial pictures expression emotions as a function of group (control vs. antibiotics) and intensity (low vs. moderate vs. high) of expressed emotion.

Mean \pm Standard error.

Group

Control Antibiotics

\begin{tabular}{lllllllll} 
& Low & Moderate & High & Average & Low & Moderate & High & Average \\
\hline Anger & $9.5 \pm 2.1$ & $56.9 \pm 6.2$ & $78.9 \pm 4.6$ & $48.4 \pm 3.5$ & $9.8 \pm 2.4$ & $45.2 \pm 7.1$ & $83.3 \pm 5.2$ & $46.1 \pm 4.0$ \\
Disgust & $14.4 \pm 2.7$ & $83.5 \pm 4.3$ & $88.2 \pm 4.3$ & $62.0 \pm 3.0$ & $18.8 \pm 3.1$ & $77.4 \pm 4.9$ & $83.1 \pm 4.9$ & $59.8 \pm 3.5$ \\
Fear & $11.4 \pm 1.8$ & $85.5 \pm 3.0$ & $93.6 \pm 2.3$ & $63.5 \pm 1.7 *$ & $7.1 \pm 2.1$ & $71.5 \pm 3.4$ & $91.0 \pm 2.6$ & $56.5 \pm 1.9^{*}$ \\
Happy & $23.5 \pm 3.9$ & $85.7 \pm 3.8$ & $96.6 \pm 1.5$ & $68.6 \pm 2.5$ & $16.0 \pm 4.4$ & $81.6 \pm 4.3$ & $94.9 \pm 1.7$ & $64.2 \pm 2.8$ \\
Sad & $15.7 \pm 4.1$ & $58.8 \pm 6.9$ & $67.5 \pm 7.8$ & $47.3 \pm 5.5$ & $13.0 \pm 4.7$ & $39.9 \pm 7.8$ & $67.5 \pm 8.9$ & $40.1 \pm 6.2$ \\
Average & $14.9 \pm 1.4$ & $74.1 \pm 3.0^{*}$ & $85.0 \pm 2.7$ & $58.0 \pm 2.1$ & $12.9 \pm 1.7$ & $63.1 \pm 3.5^{*}$ & $84.0 \pm 3.1$ & $53.3 \pm 2.4$
\end{tabular}

$* p<.05$

Reaction times

RM ANOVA on RTs resulted in listwise exclusion of 18 participants because of 0 percent accuracy scores in the low intensity emotion category. To prevent deletion of such a large number of cases, given that between-group accuracy differences were reported disregarding intensity or on the moderate intensity emotions specifically, we therefore excluded the low intensity category from further analyses. Hence, RM ANOVA on RTs was performed on moderate and high intensity emotions only, resulting in inclusion of 27 cases (12 antibiotics vs. 15 control). This revealed two significant sources of variance. First, a main effect of emotion, $F(3.211,80.269)=10.204, p<.001, \eta_{\mathrm{p}}{ }^{2}=.290$. Participants were fastest in recognizing anger $(M=990 \mathrm{~ms}, S E=78 \mathrm{~ms})$, followed by happiness $(M=1048 \mathrm{~ms}, S E=60 \mathrm{~ms})$, disgust $(M=1076 \mathrm{~ms}, S E=95 \mathrm{~ms})$, fear $(M=1322 \mathrm{~ms}, S E=83 \mathrm{~ms})$, and sadness $(M=1483 \mathrm{~ms}, S E=$ 113ms). Secondly, we found a main effect of intensity, $F(1,25)=7.395, p=.012, \eta_{\mathrm{p}}{ }^{2}=.228$, demonstrating participants were faster in accurately recognizing high intensity $(M=1124 \mathrm{~ms}, S E=$ $72 \mathrm{~ms})$ as compared to moderate intensity $(M=1243 \mathrm{~ms}, S E=64 \mathrm{~ms})$ emotions. 


\section{Discussion}

The gut microbiome shows a bidirectional relation with the brain, which may have implications for psychological and cognitive outcome measures, but such implications remain mainly untested (Sarkar et al., 2018). Dysbiosis of the gut microbiome, impacting functioning of the microbiota-gutbrain axis, has been linked to psychiatric disorders such as depression, anxiety, autism, schizophrenia, and Parkinson's (Sternbach and State, 1997; Jiang et al., 2018; Iovene et al., 2017; Castro-Nallar et al., 2015; Lin et al., 2018; Sarkar et al., 2018) including a high comorbidity between psychiatric disorders and gastrointestinal disorders (Rogers et al., 2016). As the intake of antibiotics has shown a steep increase in the past decade (Haesker et al., 2012) and is known to result in dysbiosis of the gut microbiome (Nicholson et al., 2012; Rieder et al., 2017), research on its consequences for mental functioning and well-being may be considered valuable, also in light of gaining further insight on functioning of the microbiota-gut-brain axis and the targeting potential it may provide for the prevention and treatment of stress-related disorders.

In the current study, we investigated the association between antibiotic use and cognitive reactivity to sad mood, as well as the ability to recognize other's facial emotion expressions. Regarding cognitive reactivity, we found antibiotic treatment was associated with more aggressive thought patterns in response to sad mood. Considering the findings of Steenbergen et al. (2015), reporting a decrease in aggressive and ruminative cognitions in response to sad mood following a 4-week probiotic intervention, our results confirm the idea that antibiotics may exert opposing effects on cognitive reactivity. No differences between the two groups were found regarding depression symptoms, current mood state, or other forms of cognitive reactivity (e.g. rumination).

Regarding facial emotion expression recognition, antibiotic treatment was associated with decreased recognition accuracy for moderate intensity emotional expressions; compared to individuals not having taken any antibiotic treatment in the past year, individuals that reported to have finished antibiotic treatment in the past month were less able to correctly identify an emotional facial expression when there was some ambiguity. This is in line with earlier findings, demonstrating that moderate- 
intensity facial expression of emotions are most sensitive for detecting differences (Labuschagne et al., 2010). In addition, we found preliminary support for the idea that antibiotic treatment was associated with a decreased ability to recognize fear, independent of expression intensity.

Given the correlational nature of the current study, its small sample size, and possible heterogeneity within the antibiotic group regarding type, dosage, and duration of, as well as the reason for receiving, antibiotic treatment, we can only speculate with regard to explanations of the aforementioned results. However, our results do support the idea that antibiotics might increase vulnerability for stress-related disorders, especially depression, through increasing cognitive reactivity to sad mood as well as impairing one's ability to correctly recognize emotions; factors known to increase depression vulnerability (Harmer et al., 2001; Segal et al., 1999; van der Does, 2002; Segal et al., 2006). Our results also allow antidepressive effects of antibiotics, especially regarding emotional reactivity in people suffering from depressive symptoms; typically showing enhanced sensitivity for, i.e. recognition of, negative emotional expressions (for a review see Leppänen, 2006). Noteworthy, the observed effects may reflect changes in cognitive and emotional reactivity that are particularly socially relevant. That is, aggression is an emotion typically acted upon towards others and recognizing emotions of others is essential for positive social interaction; our findings may point at the importance of further investigating particularly socially relevant reactions that may be altered by alterations of the gut microbiome.

Taken together, this study is the first to associate antibiotic usage to cognitive reactivity to sad mood and the ability to recognize others emotions. Our findings contribute to the idea that the microbiota-gut-brain axis modulates cognitive- and affective processes, and possibly subsequent wellbeing. However, our findings should be taken as preliminary and call for further investigation of antibiotic, as well as probiotic, effects regarding cognitive- and emotional reactivity to stressors. 


\section{Acknowledgements}

This research was supported by an ABC talent grant awarded by the Amsterdam centre for Brain and Cognition and a Veni grant from the Netherlands Organization for Scientific research awarded to Laura Steenbergen. The authors would like to express their gratitude to Nada Amekran, Eline Hylkema, Roseline Kuper, Hannah van de Rest, and Laura Weissman, for their valuable assistance in data collection.

\section{Author Contributions and Competing Interests}

Author LS designed the study and wrote the protocol. Authors ACDdG and LS managed the literature searches, data collection, and analyses. Authors ACDdG and LS undertook the statistical analysis, and ACDdG wrote the first draft of the manuscript. Both authors contributed to and have approved the final manuscript. The authors declare no conflict of interest.

\section{References}

Bercik, P., \& Collins, S. M. (2014). The effects of inflammation, infection and antibiotics on the microbiota-gut-brain axis. In microbial endocrinology: the microbiota-gut-brain axis in health and disease (pp. 279-289). Springer, New York, NY.

Bercik, P., Denou, E., Collins, J., Jackson, W., Lu, J., Jury, J., ... \& Verdu, E. F. (2011). The intestinal microbiota affect central levels of brain-derived neurotropic factor and behavior in mice. Gastroenterology, 141(2), 599-609.

Bouma, J., Ranchor, A. V., Sanderman, R., \& Van Sonderen, E. (1995). Het meten van symptomen van depressie met de CES-D. Een handleiding. Groningen, Noordelijk Centrum voor Gezondheidsvraagstukken, Rijksuniversiteit Groningen, 2-24.

Castro-Nallar, E., Bendall, M. L., Pérez-Losada, M., Sabuncyan, S., Severance, E. G., Dickerson, F. B., ... \& Crandall, K. A. (2015). Composition, taxonomy and functional diversity of the oropharynx microbiome in individuals with schizophrenia and controls. PeerJ, 3, e1140.

Cryan, J. F., \& Dinan, T. G. (2012). Mind-altering microorganisms: the impact of the gut microbiota on brain and behaviour. Nature reviews neuroscience, 13(10), 701. 
Cryan, J. F., \& O’Mahony, S. M. (2011). The microbiome-gut-brain axis: from bowel to behavior. Neurogastroenterology \& Motility, 23(3), 187-192.

Dean, O. M., Kanchanatawan, B., Ashton, M., Mohebbi, M., Ng, C. H., Maes, M., ... \& McKenzie, H. (2017). Adjunctive minocycline treatment for major depressive disorder: a proof of concept trial. Australian \& New Zealand Journal of Psychiatry, 51(8), 829-840.

Delungahawatta, T., Amin, J. Y., Stanisz, A. M., Bienenstock, J., Forsythe, P., \& Kunze, W. A. (2017). Antibiotic driven changes in gut motility suggest direct modulation of enteric nervous system. Frontiers in neuroscience, 11, 588.

Dudek-Wicher, R. K., Junka, A., \& Bartoszewicz, M. (2018). The influence of antibiotics and dietary components on gut microbiota. Przeglad gastroenterologiczny, 13(2), 85.

Ekman, P., \& Friesen, W. V. (1976). Measuring facial movement. Environmental psychology and nonverbal behavior, 1(1), 56-75.

Fiore, M., Moroni, R., Alleva, E., \& Aloe, L. (1996). Schistosoma mansoni: influence of infection on mouse behavior. Experimental parasitology, 83(1), 46-54.

Forsythe, P., Kunze, W., \& Bienenstock, J. (2016). Moody microbes or fecal phrenology: what do we know about the microbiota-gut-brain axis?. BMC medicine, 14(1), 58.

Foster, J. A., \& McVey-Neufeld, K. A. (2013). Gut-brain axis: how the microbiome influences anxiety and depression. Trends in neurosciences, 36(5), 305-312.

Haeseker, M. B., Dukers-Muijrers, N. H., Hoebe, C. J., Bruggeman, C. A., Cals, J. W., \& Verbon, A. (2012). Trends in antibiotic prescribing in adults in Dutch general practice. PLoS One, 7(12), e51860.

Harmer, C. J. (2008). Serotonin and emotional processing: does it help explain antidepressant drug action?. Neuropharmacology, 55(6), 1023-1028.

Harmer, C. J., Perrett, D. I., Cowen, P. J., \& Goodwin, G. M. (2001). Administration of the betaadrenoceptor blocker propranolol impairs the processing of facial expressions of sadness. Psychopharmacology, 154(4), 383-389. 
Iovene, M. R., Bombace, F., Maresca, R., Sapone, A., Iardino, P., Picardi, A., ... \& de Magistris, L. (2017). Intestinal dysbiosis and yeast isolation in stool of subjects with autism spectrum disorders. Mycopathologia, 182(3-4), 349-363.

Jiang, H. Y., Zhang, X., Yu, Z. H., Zhang, Z., Deng, M., Zhao, J. H., \& Ruan, B. (2018). Altered gut microbiota profile in patients with generalized anxiety disorder. Journal of psychiatric research, 104, 130-136.

Kelly, J. R., Allen, A. P., Temko, A., Hutch, W., Kennedy, P. J., Farid, N., ... \& Clarke, G. (2017). Lost in translation? The potential psychobiotic Lactobacillus rhamnosus (JB-1) fails to modulate stress or cognitive performance in healthy male subjects. Brain, behavior, and immunity, 61, 50-59.

Kemmis, L., Hall, J. K., Kingston, R., \& Morgan, M. J. (2007). Impaired fear recognition in regular recreational cocaine users. Psychopharmacology, 194(2), 151-159.

Labuschagne, I., Phan, K. L., Wood, A., Angstadt, M., Chua, P., Heinrichs, M., ... \& Nathan, P. J. (2010). Oxytocin attenuates amygdala reactivity to fear in generalized social anxiety disorder. Neuropsychopharmacology, 35(12), 2403.

Leclercq, S., Mian, F. M., Stanisz, A. M., Bindels, L. B., Cambier, E., Ben-Amram, H., ... \& Bienenstock, J. (2017). Low-dose penicillin in early life induces long-term changes in murine gut microbiota, brain cytokines and behavior. Nature Communications, 8, 15062.

Leppänen, J. M. (2006). Emotional information processing in mood disorders: a review of behavioral and neuroimaging findings. Current opinion in psychiatry, 19(1), 34-39.

Lin, A., Zheng, W., He, Y., Tang, W., Wei, X., He, R., ... \& Xie, H. (2018). Gut microbiota in patients with Parkinson's disease in southern China. Parkinsonism \& related disorders.

Luna, R. A., \& Foster, J. A. (2015). Gut brain axis: diet microbiota interactions and implications for modulation of anxiety and depression. Current opinion in biotechnology, 32, 35-41.

Lurie, I., Yang, Y. X., Haynes, K., Mamtani, R., \& Boursi, B. (2015). Antibiotic exposure and the risk for depression, anxiety, or psychosis: a nested case-control study. The Journal of clinical psychiatry, 76(11), 1522-1528. 
Lyte, M., Li, W., Opitz, N., Gaykema, R. P., \& Goehler, L. E. (2006). Induction of anxiety-like behavior in mice during the initial stages of infection with the agent of murine colonic hyperplasia Citrobacter rodentium. Physiology \& behavior, 89(3), 350-357.

Macedo, D., Chaves Filho, A. J. M., de Sousa, C. N. S., Quevedo, J., Barichello, T., Júnior, H. V. N., \& de Lucena, D. F. (2017). Antidepressants, antimicrobials or both? Gut microbiota dysbiosis in depression and possible implications of the antimicrobial effects of antidepressant drugs for antidepressant effectiveness. Journal of affective disorders, 208, 22-32.

McVey-Neufeld, K. A., Kang, N., Bienenstock, J., \& Foster, J. A. (2011). Effects of intestinal microbiota on anxiety-like behavior. Communicative \& integrative biology, 4(4), 492-494.

Messaoudi, M., Lalonde, R., Violle, N., Javelot, H., Desor, D., Nejdi, A., ... \& Cazaubiel, J. M. (2011). Assessment of psychotropic-like properties of a probiotic formulation (Lactobacillus helveticus R0052 and Bifidobacterium longum R0175) in rats and human subjects. British Journal of Nutrition, 105(5), 755-764.

Nicholson, J. K., Holmes, E., Kinross, J., Burcelin, R., Gibson, G., Jia, W., \& Pettersson, S. (2012). Host-gut microbiota metabolic interactions. Science, 336(6086), 1262-1267.

Nolen-Hoeksema, S., \& Morrow, J. (1991). A prospective study of depression and posttraumatic stress symptoms after a natural disaster: the 1989 Loma Prieta Earthquake. Journal of personality and social psychology, 61(1), 115.

Papalini, S., Michels, F., Kohn, N., Wegman, J., van Hemert, S., Roelofs, K., ... \& Aarts, E. (2019). Stress matters: Randomized controlled trial on the effect of probiotics on neurocognition. Neurobiology of Stress, 10, 100141.

Preidis, G. A., \& Versalovic, J. (2009). Targeting the human microbiome with antibiotics, probiotics, and prebiotics: gastroenterology enters the metagenomics era. Gastroenterology, 136(6), 20152031.

Radloff, L. S. (1977). The CES-D scale: A self-report depression scale for research in the general population. Applied psychological measurement, 1(3), 385-401. 
Raes, F., Daems, K., Feldman, G. C., Johnson, S. L., \& Van Gucht, D. (2009a). A psychometric evaluation of the Dutch version of the responses to positive affect questionnaire. Psychologica Belgica, 49(4), 293.

Raes, F., Schoofs, H., Hoes, D., Hermans, D., Van Den Eede, F., \& Franck, E. (2009b). Reflection en brooding als subtypes van rumineren: een herziening van de Ruminative Response Scale. Gedragstherapie, 42, 205-214. (Nederlandstalige versie)

Rhee, S. H., Pothoulakis, C., \& Mayer, E. A. (2009). Principles and clinical implications of the brain-gut-enteric microbiota axis. Nature reviews Gastroenterology \& hepatology, 6(5), 306.

Rieder, R., Wisniewski, P. J., Alderman, B. L., \& Campbell, S. C. (2017). Microbes and mental health: a review. Brain, behavior, and immunity.

Rogers, G. B., Keating, D. J., Young, R. L., Wong, M. L., Licinio, J., \& Wesselingh, S. (2016). From gut dysbiosis to altered brain function and mental illness: mechanisms and pathways. Molecular psychiatry, 21(6), 738 .

Sarkar, A., Harty, S., Lehto, S. M., Moeller, A. H., Dinan, T. G., Dunbar, R. I., ... \& Burnet, P. W. (2018). The microbiome in psychology and cognitive neuroscience. Trends in cognitive sciences.

Segal, Z. V., Gemar, M., \& Williams, S. (1999). Differential cognitive response to a mood challenge following successful cognitive therapy or pharmacotherapy for unipolar depression. Journal of Abnormal Psychology, 108(1), 3.

Segal, Z. V., Kennedy, S., Gemar, M., Hood, K., Pedersen, R., \& Buis, T. (2006). Cognitive reactivity to sad mood provocation and the prediction of depressive relapse. Archives of general psychiatry, 63(7), 749-755.

Sheehan, D.V., Lecrubier, Y., Sheehan, K.H., Amorim, P., Janavs, J., Weiller, E., Hergueta, T., Baker, R., Dunbar, G.C. (1998). The Mini-International Neuropsychiatric Interview (M.I.N.I.): the development and validation of a structured diagnostic psychiatric interview for DSM-IV and ICD10. J. Clin. Psychiatry, 59,22-33 
Sherwin, E., Dinan, T. G., \& Cryan, J. F. (2018). Recent developments in understanding the role of the gut microbiota in brain health and disease. Annals of the New York Academy of Sciences, 1420(1), $5-25$.

Slykerman, R. F., Thompson, J., Waldie, K. E., Murphy, R., Wall, C., \& Mitchell, E. A. (2017). Antibiotics in the first year of life and subsequent neurocognitive outcomes. Acta Paediatrica, 106(1), 87-94.

Solis, E., Antypa, N., Conijn, J. M., Kelderman, H., \& Van der Does, W. (2017). Psychometric properties of the Leiden Index of Depression Sensitivity (LEIDS). Psychological assessment, 29(2), 158.

Steenbergen, L., Sellaro, R., van Hemert, S., Bosch, J. A., \& Colzato, L. S. (2015). A randomized controlled trial to test the effect of multispecies probiotics on cognitive reactivity to sad mood. Brain, behavior, and immunity, 48, 258-264.

Sternbach, H., \& State, R. (1997). Antibiotics: neuropsychiatric effects and psychotropic interactions. Harvard review of psychiatry, 5(4), 214-226.

Tillisch, K., Labus, J., Kilpatrick, L., Jiang, Z., Stains, J., Ebrat, B., ... \& Mayer, E. A. (2013). Consumption of fermented milk product with probiotic modulates brain activity. Gastroenterology, 144(7), 1394-1401.

Turetsky, B. I., Kohler, C. G., Indersmitten, T., Bhati, M. T., Charbonnier, D., \& Gur, R. C. (2007). Facial emotion recognition in schizophrenia: when and why does it go awry?. Schizophrenia research, 94(1-3), 253-263.

Van der Does, A. J. W., \& Williams, J. M. G. (2003). Leiden index of depression sensitivity-revised (LEIDS-R). Leiden University.

Van der Does, W. (2002). Cognitive reactivity to sad mood: structure and validity of a new measure. Behaviour Research and Therapy, 40(1), 105-119.

Wallace, C. J., \& Milev, R. (2017). The effects of probiotics on depressive symptoms in humans: a systematic review. Annals of general psychiatry, 16(1), 14.

Wang, H., Braun, C., \& Enck, P. (2018). Effects of Rifaximin on Central Responses to Social Stress-a Pilot Experiment. Neurotherapeutics, 1-12. 
Wang, T., Hu, X., Liang, S., Li, W., Wu, X., Wang, L., \& Jin, F. (2015). Lactobacillus fermentum NS9 restores the antibiotic induced physiological and psychological abnormalities in rats. Beneficial microbes, 6(5), 707-717.

Watson, D., Clark, L. A., \& Tellegen, A. (1988). Development and validation of brief measures of positive and negative affect: the PANAS scales. Journal of personality and social psychology, 54(6), 1063.

Zarrinpar, A., Chaix, A., Xu, Z. Z., Chang, M. W., Marotz, C. A., Saghatelian, A., ... \& Panda, S. (2018). Antibiotic-induced microbiome depletion alters metabolic homeostasis by affecting gut signaling and colonic metabolism. Nature communications, 9(1), 2872. 